\title{
Implementation of Humane Endpoints in a Urinary Bladder Carcinogenesis Study in Rats
}

\author{
MÓNICA OLIVEIRA ${ }^{1}$, ELISABETE NASCIMENTO-GONÇALVES ${ }^{2}$, JESSICA SILVA ${ }^{2}$, PAULA A. OLIVEIRA ${ }^{2,3}$, \\ RITA FERREIRA $^{4}$, LUÍS ANTUNES ${ }^{2,3}$, REGINA ARANTES-RODRIGUES $^{3,5}$ and ANA I. FAUSTINO-ROCHA ${ }^{3,6}$ \\ ${ }^{1}$ School of Life Sciences and Environment, \\ University of Trás-os-Montes and Alto Douro (UTAD), Vila Real, Portugal; \\ ${ }^{2}$ Department of Veterinary Sciences, School of Agrarian and Veterinary Sciences, UTAD, Vila Real, Portugal; \\ ${ }^{3}$ Center for the Research and Technology of Agro-Environmental and Biological Sciences (CITAB), Vila Real, Portugal; \\ ${ }^{4}$ Organic Chemistry, Natural Products and Foodstuffs (QOPNA), \\ Mass Spectrometry Center, Department of Chemistry, University of Aveiro, Aveiro, Portugal; \\ ${ }^{5}$ Cytogenetic Laboratory, Hospital Center of Trás-os-Montes and Alto Douro, Vila Real, Portugal; \\ ${ }^{6}$ Faculty of Veterinary Medicine, Lusophone University of Humanities and Technologies (ULHT), Lisbon, Portugal
}

\begin{abstract}
Background/Aim: This study aimed to evaluate the utility of several biological parameters for the prediction of tumor development and animal welfare in a rat model of urinary bladder cancer. Materials and Methods: The control group $(n=9)$ received tap water while the test group $(n=12)$ received the carcinogen $N$-butyl-N-(4-hydroxybutyl) nitrosamine (BBN) in drinking water. A score sheet with biological variables was used to monitor animals' welfare. Body weight, food and drink consumption and rectal temperature were measured weekly. Blood and urine samples were collected. Results: Animals from the control group exhibited a slightly higher body weight and body weight gain. The final urine volume was higher in $B B N$ group $(p<0.05)$. All animals from the BBN group exhibited macroscopic hematuria at 35th week. Four animals were anemic in the last week of the experiment. Conclusion: The routine control of hematuria was a useful non-invasive biomarker of disease progression that may be used as a potential earlier humane endpoint. Animals did not show clinical signs of suffering that justified their sacrifice before the end of the study.
\end{abstract}

Over the last years animal models have been essential to understand the growth and subsistence of tumors, leading to

This article is freely accessible online.

Correspondence to: Ana I. Faustino-Rocha, Center for Research and Technology of Agro-Environment and Biological Sciences, University of Trás-os-Montes and Alto Douro, 5001-911, Vila Real, Portugal. Tel: +351 259350000, e-mail: anafaustino.faustino@sapo.pt

Key Words: BBN, animal models, rodents, animal welfare. the development of new and more effective therapies capable to prevent and treat cancer. Although the scientific community is concerned about the use of animals in experimental studies, they have been widely employed in cancer studies (1-4), since alternatives are not available. Concerned with the inflicted suffering in animals, William Russell and Rex Burch presented the 3R's in 1959, proposing the replacement of animals by in vitro methods, the reduction in number of animals used, and the introduction of refinements to decrease the incidence or severity of procedures in research animals (5). Although replacement and reduction are readily applied concepts, the refinement of procedures to reduce animals' suffering without producing a number of unwanted variables to the research, represents a bigger challenge.

Apart from being a serious ethical concern, pain and stress are undesirable features. Furthermore, they are a potential source of experimental errors as many physiological, immunological, endocrine and behavioral parameters may change as a consequence of such conditions, which ultimately might misrepresent the results of the trials $(6,7)$. Thereby, it became a legal and ethical obligation for the investigator to have the utmost care to keep animals in a state of optimal wellness $(4,8)$, once they are completely dependent on him $(9,10)$. Researchers should always aim to minimize animals' suffering, with good practices in the recognition and alleviation of pain and distress in animals, protecting animal welfare and encouraging good science (11, 12). When there is the possibility to cause animals' pain or suffering, investigators need to refine techniques and implement experimental endpoints, allowing early intervention, minimizing data loss and relieving animals' 
suffering (13). Thus, the application of appropriate and objective humane endpoints is crucial when refining in vivo cancer research.

Defining humane endpoints in cancer research can be challenging (14). According to the National Centre for the Replacement, Refinement and Reduction of Animals in Research, humane endpoints are defined as the criteria that allow early termination of experiments before animals experience significant harm while still meeting the experimental objectives (15). The Canadian Council on Animal Care (15) suggests the use of a Scoring System (Checklist) specific for each scientific procedure and species, which is based on general observations that animals might display during the experiment (16). Animals' behavior and physiological parameters should be monitored (14) and based on these predictions animals should be removed from the study when the proposed endpoints are reached (15, 17-19). A major obstacle to implement humane endpoints is a general lack of biomarkers that can be used as specific indicators of disease progression (20). Although sometimes tumor burden and disease progression can be directly measured using calipers, such as in assays of mammary carcinogenesis or in assays using xenograft models were the tumor cell lines are subcutaneously implanted $(21,22)$, many other tumors are not externally visible and consequently their growth cannot be monitored, as in the case of urinary bladder tumors.

In order to address these questions, the main goal of this study was to define humane endpoints in an experimental protocol using a rat model of chemically-induced urinary bladder cancer addressing several biological parameters.

\section{Materials and Methods}

Ethics statement. All animal procedures were performed in accordance with the European Directive 2010/63/EU and National Decree-Law $113 / 3013$ on the protection of animals used for scientific purposes. All experiments and procedures were carried out under Direção Geral de Alimentação e Veterinária, Approval no. 008961

Animals. Twenty-one male Wistar rats (Rattus norvegicus) with four weeks of age were obtained from Harlan Interfauna Inc. (Barcelona, Spain). Animals were group-housed in filter-capped polycarbonate cages (1500UEurostandard Type IV S, Tecniplast, Buguggiate, Italy) with corncob for bedding (Mucedola, Settimo Milanese, Milan, Italy), under controlled conditions of light/dark cycle $(12 \mathrm{~h} / 12 \mathrm{~h})$, temperature $\left(22^{\circ} \mathrm{C} \pm 2^{\circ} \mathrm{C}\right)$ and relative humidity $(50 \pm 10 \%)$. All animals had free access to a standard diet $\left(4 \mathrm{RF} 21^{\circledR}\right.$, Mucedola, Settimo Milanese, Milan, Italy) and tap water was supplied through capped water bottles (ACBT0502, Tecniplast, Buguggiate, Italy) ad libitum throughout the study. Cages were cleaned and water was changed once per week.

Experimental protocol. After one week of acclimatization, the rats were randomly divided into two groups: control $(\mathrm{n}=9)$ and $N$-butyl-
$N$-(4-hydroxybutyl) nitrosamine $(\mathrm{BBN})(\mathrm{n}=12)$. Animals from control group only received tap water along the experimental protocol. Animals from BBN group drank water with BBN (Tokyo Kasei Kogyo, Tokyo, Japan) at a concentration of $0.05 \%$, during the first 20 weeks of the experimental protocol. After this period, animals were maintained with simple water until the end of the experiment (Figure 1).

Score sheet. A form for registration of biological variables of each animal, such as body weight, body condition, mental status, coat and grooming, eyes, ears and whiskers, skin and mucosa, response to manipulation, breathing, hydration status, body temperature, urine color and urine volume, was elaborated by our team based on previous studies in animal welfare $(9,17)$ (Table I). A numerical value was attributed for each parameter, and the values' sum was used to determine the endpoints. The animals were daily observed and body weight, food and drink consumption and rectal temperature, were measured weekly. Food and water consumption were determined using a toploading scale (METTLER PM 4000, LabWrench, Midland, Canada). Mean food consumption for each animal placed in a cage in a group of five animals was calculated as the difference between the weight of the food container at the beginning of the week and the weight of food container at the end of the week, divided by the number of animals in the cage times the number of days. Mean water consumption for each animal was calculated as the difference between the weight of the water bottle at the beginning of the week and the weight of the water bottle at the end of the week, divided by the number of animals in the cage times the number of days (23). The following formula was used to calculate the body weight gain (\%): (Final body weight-Initial body weight)/Final body weight $\times 100$ (21). Animals were daily observed and the data was weekly filled in the scoring sheet.

Urine and blood collection. Urine collection was performed in several time points along the experimental protocol as suggested by Talhada and collaborators (24). For this, animals were placed in metabolic cages during $24 \mathrm{~h}$. After this period of time, urine from each animal was collected; the volume was measured and urine was macroscopically evaluated to detect the presence of hematuria. The degree of hematuria of each animal was scored with a value from 0 to 5 , where the number 0 corresponded to the absence of hematuria and 5 corresponded to severe hematuria. Blood samples (150 to $200 \mu \mathrm{l}$ per animal) were taken in several time points along the experimental protocol to microhematocrit tubes (Hirschmann ${ }^{\circledR}$ Laborgerate, Eberstadt, Germany). The last blood samples were taken by cardiac puncture at the time of animals' sacrifice. After each blood collection, microhematocrit was measured, the values were evaluated according to the reference values for the specific species defined by Havenaar et al. (25).

Animals' sacrifice. Thirty-five weeks after the start of the protocol, animals were sacrificed by intraperitoneal overdose of sodium pentobarbital (Eutasil, CEVA, Libourne, France): when deeply anaesthetized animals underwent exsanguination by cardiac puncture as indicated by Federation for Laboratory Animal Science Associations (26). A complete necropsy was carried out in all animals and all organs (heart, lungs, liver, kidneys, spleen and urinary bladder) were collected and macroscopically evaluated. The urinary bladder of each animal was fixated in situ with buffered formalin and collected as described by Teixeira et al. (27) for further histopathological evaluation. 

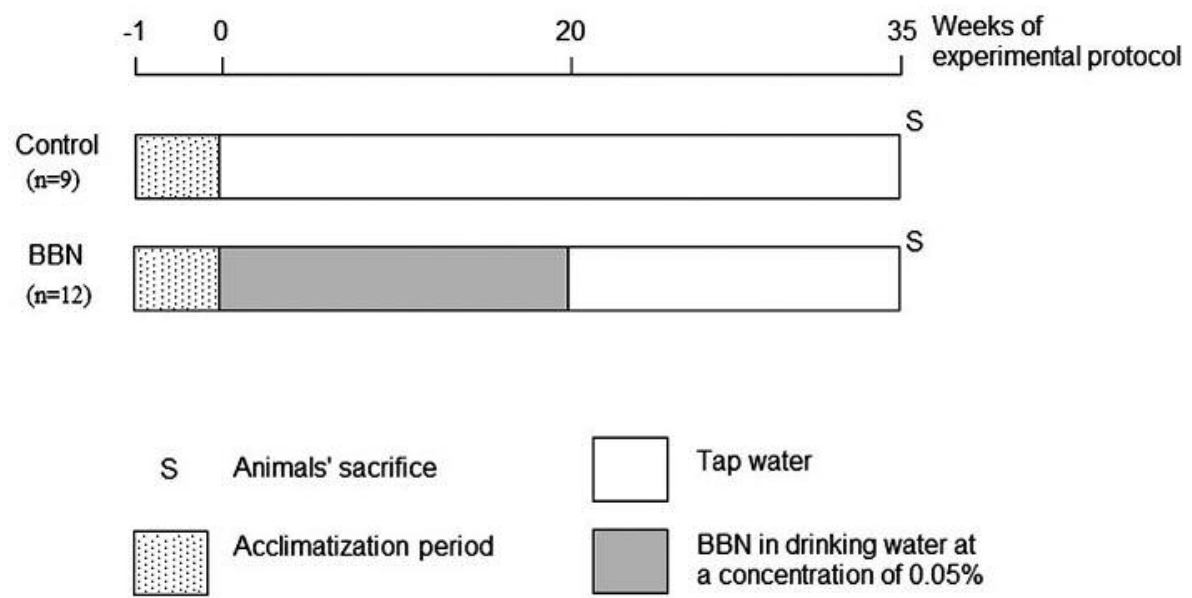

Figure 1. Experimental protocol.

Histological analysis. After fixation, urinary bladders from both control and BBN groups were processed for routine histological evaluation. Paraffin 2- $\mu \mathrm{m}$-tick sections were stained with hematoxylin and eosin (H\&E) and histologically evaluated under a light microscope by an experienced researcher (Oliveira PA) taking into account the lesion with the highest histological grade, following the WHO classification for urinary bladder tumors (28).

Statistical analysis. The statistical analysis was performed by SPSS ${ }^{\circledR}$ program (Statistical Package for the Social Sciences Inc., Chicago, IL, USA). Data were analyzed using the independent sample $t$-test. Differences between groups were tested using the independent $t$-test. Analysis of variance (ANOVA) with the Bonferroni correction test was used to evaluate the variables throughout the study. Data are showed as mean \pm standard deviation (S.D.). The differences were considered statistically significant at $p<0.05$.

\section{Results}

General observations. No deaths were recorded during the experimental protocol. All animals displayed a mental status, eyes, ears and whiskers position, response to handling, breathing and hydration within the parameters considered normal for the species. BBN-exposed animals exhibited pale mucous membranes in the last two weeks of the experimental protocol. No animal showed signs of pain or distress that implied their sacrifice before the end of the study.

Food and drink intake. We compared the mean food and water intake for control rats and for rats receiving $\mathrm{BBN}$ as measured at the end of the first week of the animals' arrival in the facility, and at the end of the study. The food intake was similar between groups $(p>0.05)$, with an initial mean consumption of $17.11 \pm 0.32 \mathrm{~g}$ and $16.89 \pm 0.90 \mathrm{~g}$ for control and $\mathrm{BBN}$ groups, respectively. A final consumption of $22.02 \pm 2.81 \mathrm{~g}$ (control group) and $21.95 \pm 0.37 \mathrm{~g}$ (BBN group) was registered $(p>0.05)$. An initial drink intake of $28.27 \pm 2.72 \mathrm{~g}$ (control group) and $25.66 \pm 1.51 \mathrm{~g}$ (BBN group) was noticed $(p>0.05)$. The final drink intake was higher in BBN group $(40.75 \pm 1.52 \mathrm{~g})$ when compared with control group $(34.08 \pm 5.14 \mathrm{~g})$, this difference was not statistically significant $(p>0.05)$.

Body weight. The animals from control group exhibited a slightly higher final body weight $(477.24 \pm 39.89 \mathrm{~g}$ versus $473.52 \pm 48.90 \mathrm{~g})$ and body weight gain $(71.26 \pm 3.10 \%$ versus $71.24 \pm 2.18 \%$ ) when compared with animals from $\mathrm{BBN}$ group. However, the differences did not reach the level of statistical significance $(p>0.05)$. According to the score sheet, there was no loss of body weight, score of 0 for this parameter for all animals.

Body temperature. During the experimental protocol, no differences were observed in rectal temperature between groups $(p>0.05)$. An initial rectal temperature of $35.98 \pm 1.04^{\circ} \mathrm{C}$ (control group) and $36.13 \pm 1.24^{\circ} \mathrm{C}(\mathrm{BBN}$ group), and a final rectal temperature of $37.06 \pm 0.37^{\circ} \mathrm{C}$ (control group) and $37.17 \pm 0.62^{\circ} \mathrm{C}(\mathrm{BBN}$ group) were registered.

Urine analysis. Although the urine volume at the beginning of the experiment was similar between groups $(14.06 \pm 5.24 \mathrm{ml}$ for control group and $14.88 \pm 5.34 \mathrm{ml}$ for BBN group) $(p>0.05)$, the final urine volume was higher in BBN group when compared with the control one $(21.33 \pm 11.42 \mathrm{ml}$ versus $13.78 \pm 1.92 \mathrm{ml})$ $(p<0.05)$. The urine from control animals did not show any macroscopic changes throughout the experimental protocol, it exhibited a clear yellow color. The urine of animals from BBN group exhibited a clear yellow color during the first 14 weeks of the experiment. Hematuria was observed for the first time at the 15 th week of the experimental protocol in an animal from the 
in vivo $31: 1073-1080(2017)$

Table I. Scoring sheet for urinary bladder cancer chemically-induced by N-butyl-N-(4-hydroxybutyl) nitrosamine (BBN).

\begin{tabular}{|c|c|c|}
\hline Parameters & Score & Status \\
\hline \multirow{5}{*}{ Body } & & Body weight \\
\hline & 0 & Normal \\
\hline & 1 & Weight loss $<10 \%$ \\
\hline & 2 & Weight loss $10-15 \%$ \\
\hline & 3 & Weight loss $>15 \%$ \\
\hline \multirow{23}{*}{ General appearance } & & Body condition \\
\hline & 0 & Well-conditioned \\
\hline & 1 & Under conditioned \\
\hline & 2 & Emaciated \\
\hline & & Mental status \\
\hline & 0 & Normal (alert, curious, eyes bright) \\
\hline & 1 & Lethargic \\
\hline & 2 & Stupor \\
\hline & 3 & Moribund / Coma \\
\hline & & Coat and grooming \\
\hline & 0 & Normal \\
\hline & 1 & Lack of grooming \\
\hline & 2 & Rough coat, chromodachryorrhea \\
\hline & 3 & Very rough coat, piloerection, severe chromodachryorrhea \\
\hline & & Eyes, ears and whiskers \\
\hline & 0 & Normal \\
\hline & 1 & Partially closed eyes, droopy ears, forward whiskers \\
\hline & 2 & Completely closed eyes, droopy and curved ears, forward and bunched whiskers \\
\hline & & Skin (ears, hands and feet) and mucosa (nasal and oral) (if anemic see microhematocri \\
\hline & 0 & Normal \\
\hline & 1 & Mild Anemic \\
\hline & 2 & Moderate Anemic \\
\hline & 3 & Severe Anemic \\
\hline
\end{tabular}

\section{Response to manipulation}

Normal

Stress response to manipulation (signs of discomfort, vocalization)

Behavior

Absence of response (lethargic animal)

\section{Breathing}

Normal

Tachypnea

Hydration status (skin pinch test)

Normal

Abnormal

Clinical signs

1

0

0

2

$\begin{array}{cc} & 0 \\ \text { Urine } & 1 \\ & 2 \\ & 3 \\ & 4 \\ 5 \\ \\ \\ \\ \\ \\ \\ 1 \\ 2\end{array}$

\section{Body temperature}

Normal $\left(35.6-38.9^{\circ} \mathrm{C}\right)$

Hyperthermia $\left(>38.9^{\circ} \mathrm{C}\right)$

Hypothermia $\left(<35.6^{\circ} \mathrm{C}\right)$

\section{Urine color}

Normal

Hematuria (+)

Hematuria $(++)$

Hematuria (+++)

Hematuria $(++++)$

Hematuria $(+++++)$

Urine volume

Normal

Oliguria

Anuria 
BBN group. After this, more animals showed hematuria and at the 35th week all animals from BBN group exhibited hematuria (urine from all of them showed a red color) (Table II).

Microhematocrit. The microhematocrit values can be consulted in Table III. Except in weeks 0 and 3, the microhematocrit values were lower in animals from the BBN group when compared with the control group. Statistically significant differences were only found between groups at the beginning (week 0) and at weeks 8,29 and $35(p<0.05)$. It is worth to note that the hematocrit value increased between the first and the last week of the experiment in control group and the inverse was observed in BBN-exposed animals $(p<0.05)$. The degree of hematuria and the mean values of microhematocrit of each animal from BBN group in the last week of the experiment can be seen in Table IV. According to the reference values indicated by Havenaar et al. (25), four animals were considered anemic in the last week of the experiment.

Tumors induction and histological analysis. The urinary bladder of animals from control group and the remaining organs from both experimental groups did not show any macroscopic alteration. At the end of the experiment, all animals from BBN group developed urinary bladder tumors (100\% of incidence), exhibiting different levels of development that were classified as different histological grade. Two animals presenting invasive carcinoma and two animals with high-grade papillary urothelial carcinoma were those with the higher degree of hematuria and lower microhematocrit (Table IV).

\section{Discussion}

Urinary bladder cancer remains a major concern due to its high rates of incidence and mortality (29). Since chemical carcinogens were discovered, several studies were carried out with rats and mice in the last twenty years in order to analyze the effectiveness of antineoplastic drugs in urinary bladder tumors chemically-induced or implanted (1). However, the animal welfare is often neglected, and the studies reporting the occurrence of pain and stress in animals used as cancer models are rare $(30,31)$. To overcome the lack of studies in this area, this work intended to implement humane endpoints in a rat model of urinary bladder cancer chemically-induced by BBN. This model is widely used by investigators to study the pathophysiology of this disease due to their similarities to human urinary bladder cancer (32).

According to our knowledge this is the first study that examined a rat male model of BBN-induced urinary bladder tumors during a long period of time (35 weeks). Since urinary bladder cancer is more frequent in men than in women, male rats were used in our protocol (29). BBN was administered orally in drinking water at a concentration of $0.05 \%$, since it
Table II. Number of animals from $\mathrm{N}$-butyl-N-(4-hydroxybutyl) nitrosamine $(B B N)$ group with hematuria during the experimental protocol.

\begin{tabular}{lc}
\hline Time (weeks) & Number of animals with hematuria $(\mathrm{n}=12)$ \\
\hline 5 & 0 \\
15 & 1 \\
20 & 5 \\
24 & 11 \\
29 & 10 \\
35 & 12 \\
\hline
\end{tabular}

Table III. Microhematocrit values (\%) (mean \pm S.D.) in both control and $N$-butyl-N-(4-hydroxybutyl) nitrosamine (BBN) groups throughout the experimental protocol.

\begin{tabular}{lcc}
\hline Time (weeks) & \multicolumn{2}{c}{ Group } \\
\cline { 2 - 3 } & Control & BBN \\
\hline 0 & $43.25 \pm 2.25$ & $47.58 \pm 2.91^{*}$ \\
3 & $48.89 \pm 1.45$ & $49.42 \pm 2.07$ \\
8 & $51.33 \pm 1.87$ & $49.67 \pm 1.07^{\dagger}$ \\
14 & $52.89 \pm 2.09$ & $51.67 \pm 1.92$ \\
18 & $52.11 \pm 2.03$ & $51.83 \pm 1.64$ \\
24 & $50.44 \pm 1.33$ & $49.75 \pm 1.76$ \\
29 & $51.56 \pm 1.59$ & $48.92 \pm 2.57^{\ddagger}$ \\
35 & $48.67 \pm 2.18$ & $41.83 \pm 8.02^{\#}$ \\
\hline
\end{tabular}

${ }^{*} p=0.002 ;{ }^{\dagger} p=0.034 ;{ }^{\ddagger} p=0.010 ;{ }^{\#} p=0.014$ from control group.

was previously verified by other investigators that this administration method and dose are the most efficient in the induction of this type of tumors (33). After 35 weeks of the beginning of the experiment, all animals from BBN group developed urinary bladder tumors ( $100 \%$ of incidence). These results are in accordance with those reported in previous studies performed with male rats exposed to BBN during eight weeks and sacrificed 28 weeks after the start of the protocol $(33,34)$. Similarly to the results observed by Wanibuchi and colleagues (33), no animals died or exhibited major clinical signs during our experimental protocol.

Weight loss is a common clinical marker of disease severity. However, Franco and collaborators (20) suggested that alterations in body weight as a single indicator of disease progression may lead to misinterpretations, namely in cancer investigation due to the tumors' weight. A reliable interpretation of animals' physiological and psychological state can be achieved by combining body weight with other behavioral observations, such as food and water consumption. According to Stasiak et al. (35), food and water consumption are considered useful indicators of pain. Furthermore, Kelley and collaborators (36) suggested that its reduction could be 
in vivo $31: 1073-1080(2017)$

Table IV. Hematuria degree, microhematocrit (\%) and histological classification of urinary bladder tumors in each N-butyl-N-(4-hydroxybutyl) nitrosamine (BBN)-exposed animal in the last week of the experimental protocol.

\begin{tabular}{lccc}
\hline Animal & Hematuria degree & Microhematocrit $(\%$ & Histological classification \\
\hline 1 & 3 & 47 & High-grade papillary urothelial carcinoma \\
2 & 0 & 48 & High-grade papillary urothelial carcinoma \\
3 & 2 & 44 & High-grade papillary urothelial carcinoma \\
4 & 4 & $32^{*}$ & High-grade papillary urothelial carcinoma \\
5 & 1 & 48 & Low-grade papillary urothelial carcinoma \\
6 & 1 & 47 & High-grade papillary urothelial carcinoma \\
7 & 3 & $38^{*}$ & Invasive carcinoma \\
8 & 4 & $38^{*}$ & High-grade papillary urothelial carcinoma \\
9 & 3 & 47 & Low-grade papillary carcinoma \\
10 & 0 & 46 & Invasive carcinoma \\
11 & 5 & $22^{*}$ & Invasive carcinoma \\
12 & 1 & 45 & Invasive carcinoma \\
\hline
\end{tabular}

*Animals with anemia ${ }^{1}$ These tumors occupied the whole bladder lumen.

seen as a general non-specific sign of disease. The analysis of these three parameters as a whole, namely a reduced food and water intake combined with a noticeable decrease in body weight may have considerable negative consequences for the animals, since malnutrition may lead to cachexia, and eventually, morbidity and mortality (37). Animals from BBN group showed a lower final mean body weight when compared with animals from control group $(p>0.05)$. This is in accordance with data previously published by Iatropoulos et al. (38) and Padrão et al. (39). The body weight loss can be associated with the development of urinary bladder tumors due to the BBN exposition and to the related host responses (40). Although statistically significant differences in mean food intake were not observed in this study, animals from BBN group exhibited a slightly lower food intake.

Similar to that observed by Oliveira (41), at the end of the experiment, the mean drink intake was higher in the BBN group ( $p>0.05)$ conducing to an increase of the final urine volume $(p<0.05)$. Renal macroscopic alterations were not observed in our study, which may be justified by the maintenance of the renal function due to the rapid passage of BBN trough the kidneys when compared with the time that it is in contact with urinary bladder (42).

Franco et al. (20) suggested that changes in body weight and temperature, along with biomarkers easily measured in blood samples, should be carefully investigated as potential early predictors of death/survival. One potential disadvantage of temperature as a marker of disease progression and humane endpoint is the natural variability in body temperature. Indeed, the body temperature may be influenced by a large number of factors, including time, activity and activation of the sympathetic nervous system (43). However, unlike to that observed in breast cancer (44), differences in body temperature between $\mathrm{BBN}$ and control animals were not observed.
The animal welfare scoring sheet used in this study was predictive of the tumor aggressiveness (histological grade). As expected, the urine from control animals did not show any alteration. Inversely, the number of animals from BBN group with macroscopic hematuria was increasing throughout the study due to the development of urinary bladder tumors (45-48). At 35 weeks after the beginning of the experiment, all animals from this group presented macroscopic hematuria. Accordingly, animals with hematuria showed a lower microhematocrit value (47). In alternative to the use of hematuria as a biological parameter of tumor progression and animal welfare, more advanced methodologies, such as imaging are available to monitor tumors' growth. However, some imaging modalities require repeated anesthesia, which may affect experimental purposes and have a welfare cost. Besides that, a financial cost, specialist skills and equipment are necessary (49).

To assess the animal welfare during this experimental protocol of urinary bladder carcinogenesis, several biological variables were registered. Although alterations in physiological parameters, namely in the urine volume and color and microhematocrit values were observed, no changes in the other parameters of the score sheet that justified the animals' sacrifice before the end of the study were registered. In complement to that proposed by Morton and Griffiths (19), the decision to sacrifice the animals should not only depend on sum values, but mainly on the individual evaluation of each animal as a whole.

\section{Conclusion}

The rodent animal model and experimental procedures used in this protocol were adequate to induce urinary bladder cancer. We conclude that the score sheet and evaluated 
physiological parameters are adequate to monitor animals' welfare during an assay of urinary bladder carcinogenesis. Furthermore, this work suggests that urine evaluation is a useful parameter in urinary bladder carcinogenesis studies to determine the general health and physiological status of the animals, with the particularity of being obtained noninvasively.

\section{Acknowledgements}

This work was supported by European Investment Funds by FEDER/COMPETE/POCI - Operational Competitiveness and Internationalization Programme, under Project POCI-01-0145FEDER-006958 and National Funds by FCT - Portuguese Foundation for Science and Technology, under the project UID/AGR/04033/2013, the project PTDC/DES/114122/2009, the project PTDC/DTP-DES/6077/2014 and the grant SFRH/BPD/ 101700/2014 to Arantes-Rodrigues R.

\section{Conflicts of Interest}

The Authors declare that they have no conflicts of interest.

\section{References}

1 Arantes-Rodrigues R, Colaço A, Pinto-Leite R and Oliveira PA: In vitro and in vivo experimental models as tools to investigate the efficacy of antineoplastic drugs on urinary bladder cancer. Anticancer Res 33: 1273-1296, 2013.

2 Arentsen HC, Hendricksen K, Oosterwijk E and Witjes JA: Experimental rat bladder urothelial cell carcinoma models. World J Urol 27: 313-317, 2009.

3 Pacharinsak C and Beitz A: Animal models of cancer pain. Comp Med 58: 220-233, 2008.

4 Workman P, Aboagye EO, Balkwill F, Balmain A, Bruder G, Chaplin DJ, Double JA, Everitt J, Farningham DAH, Glennie MJ, Kelland LR, Robinson V, Stratford IJ, Tozer GM, Watson S, Wedge SR and Eccles SA: Committee of the National Cancer Research Institute, Guidelines for the welfare and use of animals in cancer research. Br J Cancer 102: 1555-1577, 2010.

5 Russell W and Burch R: The principles of humane experimental techniques. Johns Hopkins University, USA, 1959.

6 Górska P: Principles in laboratory animal research for experimental purposes. Med Sci Monit 6: 171-180, 2000.

7 Padgett DA and Glaser R: How stress influences the immune response. Trends Immunol 24: 444-448, 2003.

8 Van Zutphen L, Baumans V and Beynen A: Modelos animales. Principios La Cienc Del Anim Lab, Harlan, Espanha, pp. 211219, 1999.

9 Olsson A and Sandøe P: Experimentação animal - O Dilema Ético. Bol Biotecnol 83: 33-39, 2006.

10 Harding J, Van Hoosier G and Grieder F: The contribution of laboratory animals to medical progress. Lab Anim Sci, p. 10, 2011.

11 Institute for Laboratory Animal Research (U.S.): Committee on recognition and alleviation of distress in laboratory animals. National Academies Press, 2008.

12 Nevalainen T: Animals and welfare: regulations, alternatives and guidelines. Welf Lab Anim, Springer, Netherlands. pp. 15-22, 2007.
13 Foltz C, Ullman-Cullere M: Guidelines for assessing the health and condition of mice. Lab Anim 28: 28-32, 1999.

14 Institute for Laboratory Animal Research (U.S.). Committee on recognition and alleviation of pain in laboratory animals. National Academies Press, 2009.

15 Olfert E, Bhasin J, Latt R, Macallum E, McCutcheon K, Rainnie $\mathrm{D}$ and Schunk M: Guidelines on: choosing an appropriate endpoint in experiments using animals for research, teaching and testing. Can Counc Anim Care, 1998.

16 Morton DB: Humane endpoints in animal experimentation for biomedical research: ethical, legal and practical aspects. 5-12, 1999.

17 Inspective W: Code of practice animal experiments in cancer research. Netherlands, 1999.

18 Jones H, Oates J and Trussell B: An applied approach to the assessment of severity. In: Hum. Endpoints Anim Exp Biomed Res, pp. 22-25, 1998.

19 Morton DB and Griffiths PH: Guidelines on the recognition of pain, distress and discomfort in experimental animals and an hypothesis for assessment. Vet Rec 116: 431-436, 1985.

20 Franco NH, Correia-Neves M and Olsson IAS: How "humane" is your endpoint? - Refining the science-driven approach for termination of animal studies of chronic infection. PLoS Pathog 8: e1002399, 2012.

21 Faustino-Rocha A, Oliveira PA, Pinho-Oliveira J, TeixeiraGuedes C, Soares-Maia R, Gil da Costa R, Colaço B, Pires MJ, Colaco J, Ferreira R and Ginja M: Estimation of rat mammary tumor volume using caliper and ultrasonography measurements. Lab Anim 42: 217-224, 2013.

22 Faustino-Rocha AI, Gama A, Oliveira PA, Alvarado A, FidalgoGonçalves L, Ferreira R and Ginja M: Ultrasonography as the gold standard for in vivo volumetric determination of chemicallyinduced mammary tumors. In Vivo 30: 465-472, 2016.

23 Arantes-Rodrigues R, Henriques A, Pinto-Leite R, Faustino-Rocha A, Pinho-Oliveira J, Teixeira-Guedes C, Seixas F, Gama A, Colaço $\mathrm{B}$, Colaço A and Oliveira PA: The effects of repeated oral gavage on the health of male CD-1 mice. Lab Anim 41: 129-134, 2012.

24 Talhada D, Andrade A, Faustino-Rocha AI, Teixeira-Guedes CI, Teixeira J, Arantes-Rodrigues R, Vasconcelos-Nóbrega C, Gil da Costa R and Oliveira PA: Recommendations for urine and urinary bladder collection in chemical carcinogenesis assays with rodents. Scand J Lab Anim Sci 41: 1-8, 2015.

25 Havenaar R, Meijer J, Morton D, Ritskes-Hoitinga and Zwart P: Biología y manejo de los animals de laboratorio. Principios La Cienc Del Anim Lab, SECAL, Granada, p. 23, 1999.

26 Forbes D, Blom H, Kostomitsopulos N, Moore G and Perretta G: Euroguide: on the accommodation and care of animals used for experimental and other scientific purposes, Federation of European Laboratory Animal Science Associations, London, 2007.

27 Teixeira C, Faustino A, Talhada D, Teixeira C, Arantes R, Vasconcelos-Nóbrega C, Gil da Costa R and Oliveira PA: Colheita de urina e recolha da bexiga em ensaios experimentais de carcinogénese química em roedores, Vila Real, 2013.

28 Eble JM, Sauter G, Epstein JI and Sesterhenn IA: Pathology and genetics of tumours of the urinary system and male ganital organs. WHO, IARC Press, 2004.

29 Kirkali Z, Chan T, Manoharan M, Algaba F, Busch C, Cheng L, Kiemeney L, Kriegmair M, Montironi R, Murphy WM, Sesterhenn IA, Tachibana $\mathrm{M}$ and Weider J: Bladder cancer: epidemiology, staging and grading, and diagnosis. Urology 66: 4-34, 2005. 
30 Jacobsen KR, Jørgensen P, Pipper CB, Steffensen AM, Hau J and Abelson KSP: The utility of fecal corticosterone metabolites and animal welfare assessment protocols as predictive parameters of tumor development and animal welfare in a murine xenograft model. In Vivo 27: 189-196, 2013.

31 Hunter JE, Butterworth J, Perkins ND, Bateson $M$ and Richardson CA: Using body temperature, food and water consumption as biomarkers of disease progression in mice with E $\mu$-myc lymphoma. Br J Cancer 110: 928-934, 2014.

32 Palmeira C, Oliveira PA, Lameiras C, Amaro T, Silva VM, Lopes $\mathrm{C}$ and Santos L: Biological similarities between murine chemical-induced and natural human bladder carcinogenesis. Oncol Lett 1: 373-377, 2010.

33 Wanibuchi H, Wei M, Salim EI, Kinoshita A, Morimura K, Sudo $\mathrm{K}$ and Fukushima $\mathrm{S}$ : Inhibition of rat urinary bladder carcinogenesis by the antiangiogenic drug TNP-470. Asian Pacific J Cancer Prev 7: 101-107, 2006.

34 Shimizu H, Akasaka S, Suzuki S, Akimoto M and Shimada T: Preferential gene transfer to BBN-induced rat bladder tumor by simple instillation of adenoviral vector. Urology 57: 579-584, 2001.

35 Stasiak KL, Maul D, French E, Hellyer PW and VandeWoude S: Species-specific assessment of pain in laboratory animals. Contemp Top Lab Anim Sci 42: 13-20, 2013.

36 Kelley KW, Bluthé RM, Dantzer R, Zhou JH, Shen WH, Johnson RW and Broussard SR: Cytokine-induced sickness behavior. Brain Behav Immun 17: 112-118, 2003.

37 Laviano A, Meguid MM and Rossi-Fanelli F: Cancer anorexia: clinical implications, pathogenesis, and therapeutic strategies. Lancet Oncol 4: 686-694, 2003.

38 Iatropoulos MJ, Williams GM, Wang CX, Brunnemann KD and Leber AP: Assessment of chronic toxicity and carcinogenicity in rats of Wingstay ${ }^{\circledR} 100$, a rubber antioxidant/antiozonant. Exp Toxicol Pathol 49: 153-165, 1997.

39 Padrão AI, Oliveira P, Vitorino R, Colaço B, Pires MJ, Márquez M, Castellanos E, Neuparth MJ, Teixeira C, Costa C, MoreiraGonçalves D, Cabral S, Duarte JA, Santos LL, Amado F and Ferreira R: Bladder cancer-induced skeletal muscle wasting: Disclosing the role of mitochondria plasticity. Int J Biochem Cell Biol 45: 1399-1409, 2013.
40 Tisdale MJ: Mechanisms of cancer cachexia. Physiol Rev 89: 381-410, 2009.

41 Oliveira PA: Cancerização experimental do urotélio do rato. Universidade de Trás-os-Montes e Alto Douro, 2006.

42 Frazier KS, Seely JC, Hard GC, Betton G, Burnett R, Nakatsuji S, Nishikawa A, Durchfeld-Meyer B and Bube A: Proliferative and nonproliferative lesions of the rat and mouse urinary system. Toxicol Pathol 40: 14-86, 2012.

43 Van Bogaert MJV, Groenink L, Oosting RS, Westphal KGC, Van der Gugten J and Olivier B: Mouse strain differences in autonomic responses to stress. Genes Brain Behav 5: 139-149, 2006.

44 Lawson RN and Chughtai MS: Breast cancer and body temperature. Can Med Assoc J 88: 68-70, 1963.

45 Messing EM, Young TB, Hunt VB, Gilchrist KW, Newton MA, Bram LL, Hisgen WJ, Greenberg EB, Kuglitsch ME and Wegenke JD: Comparison of bladder cancer outcome in men undergoing hematuria home screening versus those with standard clinical presentations. Urology 45: 387-397, 1995.

46 Messing EM and Vaillancourt A: Hematuria screening for bladder cancer. J Occup Med 32: 838-845, 1990.

47 Oteki T, Nagase S, Hirayama A, Sugimoto H, Hirayama K, Hattori K and Koyama A: Nutcracker syndrome associated with severe anemia and mild proteinuria. Clin Nephrol 62: 62-65, 2004.

48 Vasconcelos-Nóbrega C, Colaço A, Lopes C and Oliveira P: Review: BBN as an urothelial carcinogen. In Vivo 26: 727-739, 2012.

49 Sereno J: Eficácia do celecoxibe versus aspirina na prevenção, regressão do carcinoma da bexiga. Universidade da Beira Interior, 2009. 